\title{
Forced oscillation of third order nonlinear dynamic equations on time scales
}

\author{
by BAOGUO JiA (Guangzhou)
}

Abstract. Consider the third order nonlinear dynamic equation

$$
x^{\Delta \Delta \Delta}(t)+p(t) f(x)=g(t),
$$

on a time scale $\mathbb{T}$ which is unbounded above. The function $f \in C(\mathbb{R}, \mathbb{R})$ is assumed to satisfy $x f(x)>0$ for $x \neq 0$ and be nondecreasing. We study the oscillatory behaviour of solutions of $(*)$. As an application, we find that the nonlinear difference equation

$$
\Delta^{3} x(n)+n^{\alpha}|x|^{\gamma} \operatorname{sgn}(n)=(-1)^{n} n^{c},
$$

where $\alpha \geq-1, \gamma>0, c>3$, is oscillatory.

1. Introduction. Consider the third order nonlinear dynamic equation

$$
x^{\Delta \Delta \Delta}(t)+p(t) f(x)=g(t),
$$

and the second order nonlinear dynamic equation

$$
x^{\Delta \Delta}(t)+p(t) f(x)=g(t),
$$

on a time scale $\mathbb{T}$ which is unbounded above, and where $p(t), g(t)$ are realvalued, right-dense continuous functions on $\mathbb{T}$ and $p(t)$ is nonnegative but not eventually zero for large $t$. The function $f \in C(\mathbb{R}, \mathbb{R})$ is assumed to satisfy $x f(x)>0$ for $x \neq 0$ and be nondecreasing.

When $\mathbb{T}=\mathbb{R}$, the dynamic equation $(1.2)$ is the second order nonlinear differential equation

$$
x^{\prime \prime}(t)+p(t) f(x)=g(t) .
$$

In [5], James Wong studied the oscillatory behaviour of (1.3) and obtained the following

THEOREM 1.1. Assume that:

(i) There exists an $h \in C^{2}[0, \infty)$ such that $h^{\prime \prime}(t)=g(t)$ and $h(t)$ is oscillatory, i.e., it has unbounded zeros.

2010 Mathematics Subject Classification: 34K11, 39A10, 39A99.

Key words and phrases: forced oscillation, dynamic equation, time scale. 
(ii) $h(t)$ satisfies

$$
\int_{0}^{\infty} p(t) f\left(h_{+}(t)\right) d t=-\int_{0}^{\infty} p(t) f\left(h_{-}(t)\right) d t=+\infty
$$

where $h_{+}(t)=\max \{h(t), 0\}$ and $h_{-}(t)=\min \{h(t), 0\}$.

Then (1.3) is oscillatory.

In this paper, we extend this theorem to third order dynamic equations on time scales and as an application, we show that the nonlinear difference equation

$$
\Delta^{3} x(n)+n^{\alpha}|x|^{\gamma} \operatorname{sgn}(x)=(-1)^{n} n^{c}, \quad \gamma>0,
$$

is oscillatory, where $\alpha \geq-1, c>3$. This equation is the discrete analog of the differential equation $x^{\prime \prime \prime}(t)+t^{\alpha}|x|^{\gamma} \operatorname{sgn}(n)=t^{c} \sin t$.

For completeness we recall some basic results for dynamic equations and the calculus on time scales (see [1] and [2]). Let $\mathbb{T}$ be a time scale (i.e., a closed nonempty subset of $\mathbb{R}$ ) with $\sup \mathbb{T}=\infty$. The forward jump operator is defined by

$$
\sigma(t)=\inf \{s \in \mathbb{T}: s>t\},
$$

and the backward jump operator is defined by

$$
\rho(t)=\sup \{s \in \mathbb{T}: s<t\},
$$

where $\sup \emptyset=\inf \mathbb{T}$. If $\sigma(t)>t$, we say $t$ is right-scattered, while if $\rho(t)<t$ we say $t$ is left-scattered. If $\sigma(t)=t$ we say $t$ is right-dense, while if $\rho(t)=t$ and $t \neq \inf \mathbb{T}$ we say $t$ is left-dense. The graininess function $\mu$ for a time scale $\mathbb{T}$ is defined by

$$
\mu(t)=\sigma(t)-t,
$$

and for any function $f: \mathbb{T} \rightarrow \mathbb{R}$ the notation $f^{\sigma}(t)$ stands for $f(\sigma(t))$. We say that $x: \mathbb{T} \rightarrow \mathbb{R}$ is differentiable at $t \in \mathbb{T}$ provided

$$
x^{\Delta}(t):=\lim _{s \rightarrow t} \frac{x(t)-x(s)}{t-s}
$$

exists when $\sigma(t)=t$ (here by $s \rightarrow t$ it is understood that $s$ approaches $t$ in the time scale), and when $x$ is continuous at $t$ and $\sigma(t)>t$,

$$
x^{\Delta}(t):=\frac{x(\sigma(t))-x(t)}{\mu(t)} .
$$

Note that if $\mathbb{T}=\mathbb{R}$, then the delta derivative is just the standard derivative, and when $\mathbb{T}=\mathbb{Z}$ the delta derivative is just the forward difference operator. Hence our results contain the discrete and continuous cases as special cases and generalize these results to arbitrary time scales. 


\section{Lemma and main theorem}

Lemma 2.1. Suppose that there exists an $h \in C^{3}(\mathbb{T}, \mathbb{R})$ such that $h^{\Delta \Delta \Delta}(t)$ $=g(t)$ and $h(t)$ is oscillatory. Let $x(t)$ be a solution of $(1.1)$. Write $x(t)=$ $y(t)+h(t)$.

(i) If $x(t)>0$ for large $t$, then

$$
y(t)>0, \quad y^{\Delta \Delta}(t)>0 \quad \text { and } \quad y^{\Delta \Delta \Delta}(t) \leq 0, \quad \text { for large } t .
$$

(ii) If $x(t)<0$ for large $t$, then

$$
y(t)<0, \quad y^{\Delta \Delta}(t)<0 \quad \text { and } \quad y^{\Delta \Delta \Delta}(t) \geq 0, \quad \text { for large } t .
$$

REMARK. Under the hypothesis of Lemma 2.1, (1.1) can be rewritten as a homogeneous dynamic equation

$$
y^{\Delta \Delta \Delta}(t)+p(t) f(y(t)+h(t))=0 .
$$

Proof. (i) Suppose that $x(t)>0$ for large $t$. Since $p(t) \geq 0$, from (1.1) we note that $y^{\Delta \Delta \Delta}(t) \leq 0$ for large $t$. Hence $y^{\Delta \Delta}(t)$ is decreasing for large $t$. We claim that $y^{\Delta \Delta}(t) \geq 0$ for large $t$. Assume not; then there is a large $t_{1}$ such that $y^{\Delta \Delta}\left(t_{1}\right)<0$.

Since $y^{\Delta \Delta \Delta}(t) \leq 0$, we have $y^{\Delta \Delta}(t) \leq y^{\Delta \Delta}\left(t_{1}\right)<0$ for $t \geq t_{1}$. By the Mean Value Theorem (see Theorem 1.14 of [2]), we have $y^{\Delta}(t) \rightarrow-\infty$ as $t \rightarrow \infty$. So $y(t) \rightarrow-\infty$ as $t \rightarrow \infty$. But this together with $h(t)$ being oscillatory contradicts the assumption that $x(t)>0$ for large $t$.

In the following, we show that $y^{\Delta \Delta}(t)$ is eventually positive, i.e., $y^{\Delta \Delta}(t)$ $>0$ for large $t$. Suppose that $y^{\Delta \Delta}\left(t_{2}\right)=0$ for some large $t_{2}$. As $y^{\Delta \Delta \Delta}(t) \leq 0$ and $y^{\Delta \Delta}(t) \geq 0$, this means that $y^{\Delta \Delta}(t) \equiv 0$. Returning to (1.1), this would imply $p(t) \equiv 0$ for large $t$, contradicting the assumption.

So $y^{\Delta}(t)>0$ or $y^{\Delta}(t)<0$ for large $t$. Therefore $y(t)>0$ or $y(t)<0$ for large $t$.

Since $h(t)$ is oscillatory and $x(t)>0$, we have $y(t)>0$.

Similarly, (ii) also holds.

Theorem 2.2. Let $h(t)=\int_{t_{0}}^{t}\left\{\int_{t_{0}}^{s}\left[\int_{t_{0}}^{\tau} g(u) \Delta u\right] \Delta \tau\right\} \Delta s$. Assume that $h(t)$ is oscillatory and satisfies

$$
\begin{aligned}
& \int_{t_{0}}^{\infty} p(t) f\left(h_{+}(t)\right) \Delta t=+\infty, \\
& \int_{t_{0}}^{\infty} p(t) f\left(h_{-}(t)\right) \Delta t=-\infty,
\end{aligned}
$$

where $h_{+}(t)=\max \{h(t), 0\}$ and $h_{-}(t)=\min \{h(t), 0\}$. Then (1.1) is oscillatory. 
Proof. Assume that the solution $x(t)$ of (1.1) is nonoscillatory. Without loss of generality we can assume that $x(t)>0$ for large $t$. Let $x(t)=y(t)$ $+h(t)$. By Theorem 1.74 of [1], we have $h^{\Delta \Delta \Delta}(t)=g(t)$. From (1.1),

$$
y^{\Delta \Delta \Delta}(t)+p(t) f(y(t)+h(t))=0 .
$$

From Lemma 2.1, we have $y(t)>0, y^{\Delta \Delta}(t)>0$ and $y^{\Delta \Delta \Delta}(t) \leq 0, t \in$ $\left[t_{1}, \infty\right)$, for some $t_{1} \in \mathbb{T}$. Integrating $(2.3)$, we obtain

$$
y^{\Delta \Delta}(t)-y^{\Delta \Delta}\left(t_{1}\right)+\int_{t_{1}}^{t} p(s) f(y(s)+h(s)) \Delta s=0 .
$$

Since $y^{\Delta \Delta \Delta}(t) \leq 0, \lim _{t \rightarrow \infty} y^{\Delta \Delta}(t)$ exists and is finite; hence the integral in (2.4) converges as $t \rightarrow \infty$.

We note that for all $t \geq t_{1}, y(t)+h(t)>h_{+}(t)$. To see this, we write $y(t)+h(t)=y+h_{+}(t)+h_{-}(t)$ and observe that

(i) for $h_{+}(t)=0, y(t)+h(t)=y(t)+h_{-}(t)=x(t)>0=h_{+}(t)$,

(ii) for $h_{-}(t)=0, y(t)+h(t)=y(t)+h_{+}(t)>h_{+}(t)$, since $y(t)>0$.

Since $f(x)$ is nondecreasing, we have $f(y(t)+h(t)) \geq f\left(h_{+}(t)\right)$. Note that $p(t) \geq 0$. We now estimate as follows:

$$
\int_{t_{1}}^{t} p(s) f\left(h_{+}(s)\right) \Delta s \leq \int_{t_{1}}^{t} p(s) f(y(s)+h(s)) \Delta s<\infty .
$$

By applying (2.1) to (2.5), we obtain the desired contradiction.

Consider the $n$th order nonlinear dynamic equation

$$
x^{\Delta^{n}}(t)+p(t) f(x)=g(t)
$$

on a time scale $\mathbb{T}$ which is unbounded above, and where $p(t), g(t)$ are realvalued, right-dense continuous functions on $\mathbb{T}$ and $p(t)$ is nonnegative but not eventually zero for large $t, f(x)$ is a continuous and nondecreasing function of $x \in(-\infty, \infty)$, and $x f(x)>0$ for $x \neq 0$.

Similar to Lemma 2.1, we have

Lemma 2.3. Suppose that there exists an $h \in C^{n}(\mathbb{T}, \mathbb{R})$ such that $h^{\Delta^{n}}(t)$ $=g(t)$ and $h(t)$ is oscillatory. Let $x(t)$ be a solution of (1.1) and write $x(t)=y(t)+h(t)$.

(i) If $x(t)>0$ for large $t$, then

$$
y(t)>0, \quad y^{\Delta^{n-1}}(t)>0 \quad \text { and } \quad y^{\Delta^{n}}(t) \leq 0, \quad \text { for large } t .
$$

(ii) If $x(t)<0$ for large $t$, then

$$
y(t)<0, \quad y^{\Delta^{n-1}}(t)<0 \quad \text { and } \quad y^{\Delta^{n}}(t) \geq 0, \quad \text { for large } t .
$$

Using Lemma 2.3, we can generalize Theorem 2.2 to the $n$th order dynamic equation (2.6). 
THEOREM 2.4. Let $h(t)=\int_{t_{0}}^{t} \int_{t_{0}}^{\tau_{1}} \cdots \int_{t_{0}}^{\tau_{n-1}} g\left(\tau_{n}\right) \Delta \tau_{n} \Delta \tau_{n-1} \cdots \Delta \tau_{1}$. Assume that $h(t)$ is oscillatory and satisfies

$$
\int_{t_{0}}^{\infty} p(t) f\left(h_{+}(t)\right) \Delta t=+\infty, \quad \int_{t_{0}}^{\infty} p(t) f\left(h_{-}(t)\right) \Delta t=-\infty,
$$

where $h_{+}(t)=\max \{h(t), 0\}$ and $h_{-}(t)=\min \{h(t), 0\}$. Then (2.6) is oscillatory.

\section{Example}

EXAmple 3.1. Consider the third order difference equation

$$
\Delta^{3} x(n)+p(n)|x|^{\gamma} \operatorname{sgn}(n)=g(n), \quad \gamma>0,
$$

where $p(n)=n^{\alpha}, \alpha \geq-1, g(n)=(-1)^{n} n^{c}, c>3$.

We need the following interesting lemma.

Lemma 3.2. For each real number $c>3$, we have

$$
\lim _{m \rightarrow \infty} \frac{\sum_{i=1}^{m} i^{c}-\frac{m^{c+1}}{c+1}-\frac{m^{c}}{2}-\frac{c}{12} m^{c-1}}{m^{c-3}}=\frac{-c(c-1)(c-2)}{720} .
$$

Proof. By Taylor's formula, we have

$$
\begin{aligned}
& \left(1+\frac{1}{m}\right)^{a} \\
= & 1+\frac{a}{m}+\frac{a(a-1)}{2 m^{2}}+\frac{a(a-1)(a-2)}{6 m^{3}} \\
+ & \frac{a(a-1)(a-2)(a-3)}{24 m^{4}}+\frac{a(a-1)(a-2)(a-3)(a-4)}{120 m^{5}}+o\left(\frac{1}{m^{5}}\right)
\end{aligned}
$$

for any real number $a$. For $c>3$, by (3.3) and the Stolz-Cesàro Theorem (see Theorem 1.120 of [1] or Lemma 3.2 of [4]), it is easy to see that

$$
\begin{aligned}
& =\lim _{m \rightarrow \infty} \frac{(m+1)^{c}-\frac{(m+1)^{c+1}}{c+1}-\frac{(m+1)^{c}}{2}-\frac{c(m+1)^{c-1}}{12}+\frac{m^{c+1}}{c+1}+\frac{m^{c}}{2}+\frac{c m^{c-1}}{12}}{(m+1)^{c-3}-m^{c-3}} \\
& =\lim _{m \rightarrow \infty} \frac{\frac{m^{3}}{2}\left(1+\frac{1}{m}\right)^{c}-\frac{m^{4}}{c+1}\left(1+\frac{1}{m}\right)^{c+1}-\frac{c m^{2}}{12}\left(1+\frac{1}{m}\right)^{c-1}+\frac{m^{4}}{c+1}+\frac{m^{3}}{2}+\frac{c m^{2}}{12}}{\left(1+\frac{1}{m}\right)^{c-3}-1} .
\end{aligned}
$$

By (3.3), we have

$$
\begin{aligned}
\left(1+\frac{1}{m}\right)^{c}= & 1+\frac{c}{m}+\frac{c(c-1)}{2 m^{2}}+\frac{c(c-1)(c-2)}{6 m^{3}} \\
& +\frac{c(c-1)(c-2)(c-3)}{24 m^{4}}+o\left(\frac{1}{m^{4}}\right),
\end{aligned}
$$




$$
\begin{aligned}
& \left(1+\frac{1}{m}\right)^{c+1} \\
= & 1+\frac{c+1}{m}+\frac{(c+1) c}{2 m^{2}}+\frac{(c+1) c(c-1)}{6 m^{3}} \\
+ & \frac{(c+1) c(c-1)(c-2)}{24 m^{4}}+\frac{(c+1) c(c-1)(c-2)(c-3)}{120 m^{5}}+o\left(\frac{1}{m^{5}}\right),
\end{aligned}
$$

$$
\begin{aligned}
\left(1+\frac{1}{m}\right)^{c-1} & \\
& =1+\frac{c-1}{m}+\frac{(c-1)(c-2)}{2 m^{2}}+\frac{(c-1)(c-2)(c-3)}{6 m^{3}}+o\left(\frac{1}{m^{3}}\right) .
\end{aligned}
$$

Using (3.5)-(3.7) in (3.4), it follows that (3.2) holds.

Let $C_{1}:=-c(c-1)(c-2) / 720$. Given $0<\epsilon<1$, for large $m$, we have the inequalities

$$
\begin{aligned}
& \sum_{i=1}^{m} i^{c}<\frac{m^{c+1}}{c+1}+\frac{m^{c}}{2}+\frac{c m^{c-1}}{12}+\left(C_{1}+\epsilon\right) m^{c-3}, \\
& \sum_{i=1}^{m} i^{c}>\frac{m^{c+1}}{c+1}+\frac{m^{c}}{2}+\frac{c m^{c-1}}{12}+\left(C_{1}-\epsilon\right) m^{c-3} .
\end{aligned}
$$

Therefore for $t=m$, by integrating by parts we have

$$
\begin{aligned}
h(t)= & \int_{1}^{t} \int_{1}^{s} \int_{1} g(u) \Delta u \Delta \tau \Delta s \\
= & t \int_{1}^{t}\left[\int_{1}^{\tau} p(u) \Delta u\right] \Delta \tau-\int_{1}^{t} \sigma(s)\left[\int_{1}^{s} g(u) \Delta u\right] \Delta s \\
= & t^{2} \int_{1}^{t} p(u) \Delta u-t \int_{1}^{t} \sigma(\tau) p(\tau) \Delta \tau \\
& -\int_{1}^{t} \sigma(\tau) \Delta \tau \int_{1}^{t} p(u) \Delta u+\int_{1}^{t}\left[\int_{1}^{\sigma(s)} \sigma(\tau) \Delta \tau\right] g(s) \Delta s \\
= & \left(\frac{m^{2}}{2}-\frac{3 m}{2}+1\right) \sum_{i=1}^{m-1}(-1)^{i} i^{c}-\left(m-\frac{3}{2}\right) \sum_{i=1}^{m-1}(-1)^{i} i^{c+1} \\
& +\frac{1}{2} \sum_{i=1}^{m-1}(-1)^{i} i^{c+2} .
\end{aligned}
$$

Letting $m=2 k$, we get 
$(3.11) \quad h(2 k)$

$$
\begin{aligned}
& =\left(2 k^{2}-3 k+1\right) \sum_{i=1}^{2 k-1}(-1)^{i} i^{c}-\left(2 k-\frac{3}{2}\right) \sum_{i=1}^{2 k-1}(-1)^{i} i^{c+1}+\frac{1}{2} \sum_{i=1}^{2 k-1}(-1)^{i} i^{c+2} \\
& =\left(2 k^{2}-3 k+1\right) \sum_{i=1}^{2 k-2}(-1)^{i} i^{c}-\left(2 k-\frac{3}{2}\right) \sum_{i=1}^{2 k-2}(-1)^{i} i^{c+1}+\frac{1}{2} \sum_{i=1}^{2 k-2}(-1)^{i} i^{c+2}
\end{aligned}
$$

since the terms corresponding to $i=2 k-1$ cancel. From (3.8) and (3.9), it is easy to see that

$$
\sum_{i=1}^{2 k-2}(-1)^{i} i^{c}=-\sum_{i=1}^{2 k-2} i^{c}+2^{1+c} \sum_{i=1}^{k-1} i^{c}
$$

$$
\begin{aligned}
\leq & -\left[\frac{(2 k-2)^{c+1}}{c+1}+\frac{(2 k-2)^{c}}{2}+\frac{c(2 k-2)^{c-1}}{12}+\left(C_{1}-\epsilon\right)(2 k-2)^{c-3}\right] \\
& +2^{1+c}\left[\frac{(k-1)^{c+1}}{c+1}+\frac{(k-1)^{c}}{2}+\frac{c(k-1)^{c-1}}{12}+\left(C_{1}+\epsilon\right)(k-1)^{c-3}\right] \\
= & \frac{(2 k-2)^{c}}{2}+\frac{c(2 k-2)^{c-1}}{4}+\left[C_{1}\left(2^{4}-1\right)+\epsilon\left(2^{4}+1\right)\right](2 k-2)^{c-3}
\end{aligned}
$$

and

$$
\begin{aligned}
\sum_{i=1}^{2 k-2}(-1)^{i} i^{c} \geq & \frac{(2 k-2)^{c}}{2}+\frac{c(2 k-2)^{c-1}}{4} \\
& +\left[C_{1}\left(2^{4}-1\right)-\epsilon\left(2^{4}+1\right)\right](2 k-2)^{c-3} .
\end{aligned}
$$

From (3.11)-(3.13), we get

$$
\begin{aligned}
h(2 k) \leq & \left(2 k^{2}-3 k+1\right)\left[\frac{(2 k-2)^{c}}{2}+\frac{c(2 k-2)^{c-1}}{4}\right. \\
& \left.+\left[C_{1}\left(2^{4}-1\right)+\epsilon\left(2^{4}+1\right)\right](2 k-2)^{c-3}\right] \\
& -\left(2 k-\frac{3}{2}\right)\left[\frac{(2 k-2)^{c+1}}{2}+\frac{(c+1)(2 k-2)^{c}}{4}\right. \\
& \left.+\left[C_{1}\left(2^{4}-1\right)-\epsilon\left(2^{4}+1\right)\right](2 k-2)^{c-2}\right] \\
& +\frac{1}{2}\left[\frac{(2 k-2)^{c+2}}{2}+\frac{(c+2)(2 k-2)^{c+1}}{4}\right. \\
& \left.+\left[C_{1}\left(2^{4}-1\right)+\epsilon\left(2^{4}+1\right)\right](2 k-2)^{c-1}\right] \\
= & (2 k-2)^{c}\left[-\frac{1}{8}+O\left(\frac{1}{k}\right)\right] .
\end{aligned}
$$


Take $0<A<1 / 8$. Then

$$
h(2 k) \leq-A(2 k-2)^{c} \quad \text { for large } k .
$$

Letting $m=2 k+1$, from (3.10), (3.12) and (3.13) we get

$$
\begin{aligned}
(3.15) & h(2 k+1) \\
= & \left(2 k^{2}-k\right) \sum_{i=1}^{2 k}(-1)^{i} i^{c}-\left(2 k-\frac{1}{2}\right) \sum_{i=1}^{2 k}(-1)^{i} i^{c+1}+\frac{1}{2} \sum_{i=1}^{2 k}(-1)^{i} i^{c+2} \\
\geq & \left(2 k^{2}-k\right)\left[\frac{(2 k)^{c}}{2}+\frac{c(2 k)^{c-1}}{4}+\left[C_{1}\left(2^{4}-1\right)-\epsilon\left(2^{4}+1\right)\right](2 k)^{c-3}\right] \\
& -\left(2 k-\frac{1}{2}\right)\left[\frac{(2 k)^{c+1}}{2}+\frac{(c+1)(2 k)^{c}}{4}+\left[C_{1}\left(2^{4}-1\right)+\epsilon\left(2^{4}+1\right)\right](2 k)^{c-2}\right] \\
& +\frac{1}{2}\left[\frac{(2 k)^{c+2}}{2}+\frac{(c+2)(2 k)^{c+1}}{4}+\left[C_{1}\left(2^{4}-1\right)-\epsilon\left(2^{4}+1\right)\right](2 k)^{c-1}\right] \\
= & (2 k)^{c}\left[\frac{1}{8}+O\left(\frac{1}{k}\right)\right] .
\end{aligned}
$$

Take $0<B<1 / 8$. Then

$$
h(2 k+1) \geq B(2 k)^{c} \quad \text { for large } k .
$$

From (3.14) and (3.16), we deduce that for large $k$,

$$
\begin{array}{ll}
h_{+}(2 k)=0, & h_{+}(2 k+1) \geq B(2 k)^{c}, \\
h_{-}(2 k) \leq-A(2 k-2)^{c}, & h_{-}(2 k+1)=0 .
\end{array}
$$

So picking a large $K$, we have (note that $\alpha+c \gamma \geq \alpha \geq-1$ )

$$
\begin{aligned}
& \int_{K}^{\infty} p(s) f\left(h_{+}(s)\right) \Delta s \geq \sum_{k=K}^{\infty}(2 k+1)^{\alpha} B^{\gamma}(2 k)^{c \gamma}=+\infty, \\
& \int_{K}^{\infty} p(s) f\left(h_{-}(s)\right) \Delta s \leq-\sum_{k=K}^{\infty}(2 k)^{\alpha} A^{\gamma}(2 k-2)^{c \gamma}=-\infty .
\end{aligned}
$$

From (3.14) and (3.16), we conclude that $h(t)$ is oscillatory. Therefore by Theorem 2.2, all solutions of equation (3.1) are oscillatory. 
REMARK. When $c=3$, using the formulas

$$
\begin{aligned}
\sum_{i=1}^{n} i^{3} & =\frac{1}{4} n^{2}(n+1)^{2}, \\
\sum_{i=4}^{n} i^{4} & =\frac{1}{30} n(n+1)(2 n+1)\left(3 n^{2}+3 n-1\right), \\
\sum_{i=5}^{n} i^{5} & =\frac{1}{12} n^{2}(n+1)^{2}\left(2 n^{2}+2 n-1\right),
\end{aligned}
$$

by (3.11), (3.15) and a complicated calculation, we can get

$$
h(2 k)=51 k^{3}-\frac{55}{2} k^{2}-\frac{3}{2} k, \quad h(2 k+1)=k^{3}-\frac{7}{2} k^{2}-\frac{1}{2} k .
$$

It is easy to see that $h(m)$ does not satisfy the assumption of Theorem 2.2.

Acknowledgments. This work is supported by the National Natural Science Foundation of China (No. 10971232).

\section{References}

[1] M. Bohner and A. Peterson, Dynamic Equations on Time Scales: An Introduction with Applications, Birkhäuser, Boston, 2001.

[2] M. Bohner and A. Peterson (eds.), Advances in Dynamic Equations on Time Scales, Birkhäuser, Boston, 2003.

[3] B. G. Jia, Forced oscillation of second order nonlinear dynamic equations on time scales, submitted.

[4] B. G. Jia, L. Erbe and A. Peterson, Oscillation of sublinear Emden-Fowler dynamic equations on time scales, J. Difference Equations Appl. 16 (2010), 217-226.

[5] J. S. W. Wong, Second order nonlinear forced oscillations, SIAM J. Math. Anal. 19 (1988), 667-675.

Baoguo Jia

Department of Mathematics

Zhongshan University

Guangzhou, China 510275

E-mail: mcsjbg@mail.sysu.edu.cn

Received 20.10.2009

and in final form 10.12.2009 
